\title{
Exploring outdoor thermal perception—a revised model
}

\author{
Sanda Lenzholzer ${ }^{1} \cdot$ Sjerp de Vries ${ }^{2}$
}

Received: 11 September 2018 / Revised: 25 July 2019 / Accepted: 30 July 2019/Published online: 13 August 2019

(C) The Author(s) 2019

\begin{abstract}
This paper aims at extending earlier models of outdoor thermal perception by fusing new knowledge from recent literature and deriving perspectives for future research and methods from the new model. Previous models focused on physical and physiological aspects. Only recently, the psychological aspects of thermal perception received more attention, such as spatial perception. Furthermore, in recent literature on thermal perception, two time scales have been described: the short-term and the long-term thermal perception. Based on this new literature, we develop a conceptual, more comprehensive model that takes these factors into account as well. It hypothesizes how thermal sensation and psychological processes interact on the two time scales. However, to be able to describe relationships between psychological aspects of thermal perception more precisely, more research is required on the following issues: (1) influence of momentary personal references and preferences (e.g., mood), (2) influence of long-term personal references and preferences (e.g., cultural aspects, habituation), and (3) influence of the perception of the spatial environment on thermal perception. Moreover, the relation between momentary and long-term thermal perception has not been studied yet. We conclude this paper with an outlook on possible methods to study these factors.
\end{abstract}

Keywords Thermal comfort $\cdot$ Environmental psychology $\cdot$ Literature review $\cdot$ Conceptual model $\cdot$ Research agenda

\section{Introduction}

Humanity has to adapt its urban environment to the effects of rising frequency of heatwaves (Field 2014) and other climate issues such as urban wind effects (Blocken and Carmeliet 2004), especially when it comes to sustaining or creating human thermal comfort (Gosling et al. 2009). The reasons to adapt are manifold: we need to support safety, well-being, and health, maintain high work productivity, and lower the mortality rates during heat waves (Blocken and Carmeliet 2004; Haines et al. 2006; Kovats and Hajat 2008; D'Ippoliti et al. 2010). To effectively change thermal conditions and the way people perceive and appreciate them, we need better evidence about the impact of the factors we can influence. These

Sanda Lenzholzer

sanda.lenzholzer@wur.nl

Sjerp de Vries

sjerp.devries@wur.nl

1 Landscape Architecture and Spatial Planning, Wageningen University, P.O. box 47, 6700 AA Wageningen, The Netherlands

2 Wageningen Environmental Research, P.O. box 47, 6700 AA Wageningen, The Netherlands factors can concern physical/physiological and psychological aspects. In the past decades, an increasing amount of studies generated evidence with regard to the physiological factors of outdoor thermal sensation (e.g., sweat rates, metabolic response) and the physical microclimatic circumstances influencing outdoor thermal sensation. Quite recently, initial research mapped the psychological factors influencing thermal perception. In 2009, Knez and colleagues came up with a conceptual model that tried to capture the different influences on thermal perception, being "place" (e.g., physical base, weather, function), "moderator/mediator" (culture, person, situation) and "human response." This model provided a good overview of factors but it did not differentiate the role of shortand long-term perception that was described in that same paper. However, these different time scales in human perception are significant. For instance, the short-term weather conditions shape the momentary thermal perception, but the accumulated weather experience can lead to long-term perception. Also, the model did not focus on the factors that can be actively changed such as the reshaping of spatial environments. These spatial factors are increasingly relevant in the light of climate adaptation because the spatial environment has a very strong micrometeorological effect (Brown and Gillespie 1995; Lenzholzer 2015; Oke et al. 2017). Apart from that, the spatial environment has an effect on the human psychological state 
that was not mentioned before. Moreover, the relations between the concepts in the model of Knez and colleagues were rather indicative. To be able to influence thermal perception effectively, it is necessary to understand these relations in a more precise way. Hence, we deem a refinement of this earlier model necessary, taking into account time scales and making relations more explicit. To generate a revised conceptual model, we studied the literature, and based on this new conceptual model, we identify a range of knowledge gaps that need to be addressed to understand certain aspects of the model and their relationships. We conclude this paper with suggesting methods to study these underrepresented aspects.

\section{Reshaping the existing model}

To develop the extended model of outdoor thermal perception, we studied the literature with special focus on the aspects that influence short- and long-term outdoor thermal perception. Based on the most recent findings, it combined the different parameters into a new conceptual model for outdoor thermal perception (see Fig. 1). The new model entails the factors used by Knez et al. (2009) but it extends these and differentiates between the long- and short-term factors as well as the realms in which they manifest themselves: the physical/physiological and the psychological realm. We assume that the physical realm to a large extent shapes the thermal sensation and therefore positions it at the beginning of the causal chain that we extend with a distinctive part that describes the psychological realm. As this new model entails the psychological factors explicitly, we call it the "physical/physiological-psychological model for outdoor thermal perception" (PhyPsy model). In the following, we will describe the model's different parts and factors in more detail and from which sources in the literature they were derived, with a focus on the new sources covering the fields of the psychological realm.

The regional climate and weather (boxes A and B in Fig. 1) are obviously main drivers of local climate and weather. The regional climate with its stable characteristics (e.g., in the temperate maritime climate in Northwestern Europe) influences typical microclimate on a location to a large extent (box D). At the same time, microclimate is also formed by local spatial characteristics (box C) such as the shapes of built volumes and open spaces, the amount of vegetation, and water in the environment (Oke et al. 2017). However, regional weather (box B) can change quickly and together with effects of spatial configurations on a location, they result in the so-called microweather (Westerberg et al. 2003; Lenzholzer 2010; Jacobs et al. 2015) (box E). The micro-weather circumstances consist of radiation fluxes, air temperature, wind speeds, etc. (Brown and Gillespie 1995; Oke et al. 2017). To a large extent, the micro-weather governs human thermal sensation (box G) in a specific location at a specific point in time. "Thermal sensation" denotes the purely physiological part of thermal perception (Humphreys and Hancock 2007; Knez et al. 2009). Additional momentary factors that influence this sensation are amongst other clothing, a person's metabolism (depending on the type of their physical activity at that moment), and the length of exposure to the local microclimate (Parsons 2003; Cheung and Jim 2018) (box F). The thermal sensation has been expressed in various indices. Amongst the most common indices used for outdoor situations are predicted mean vote

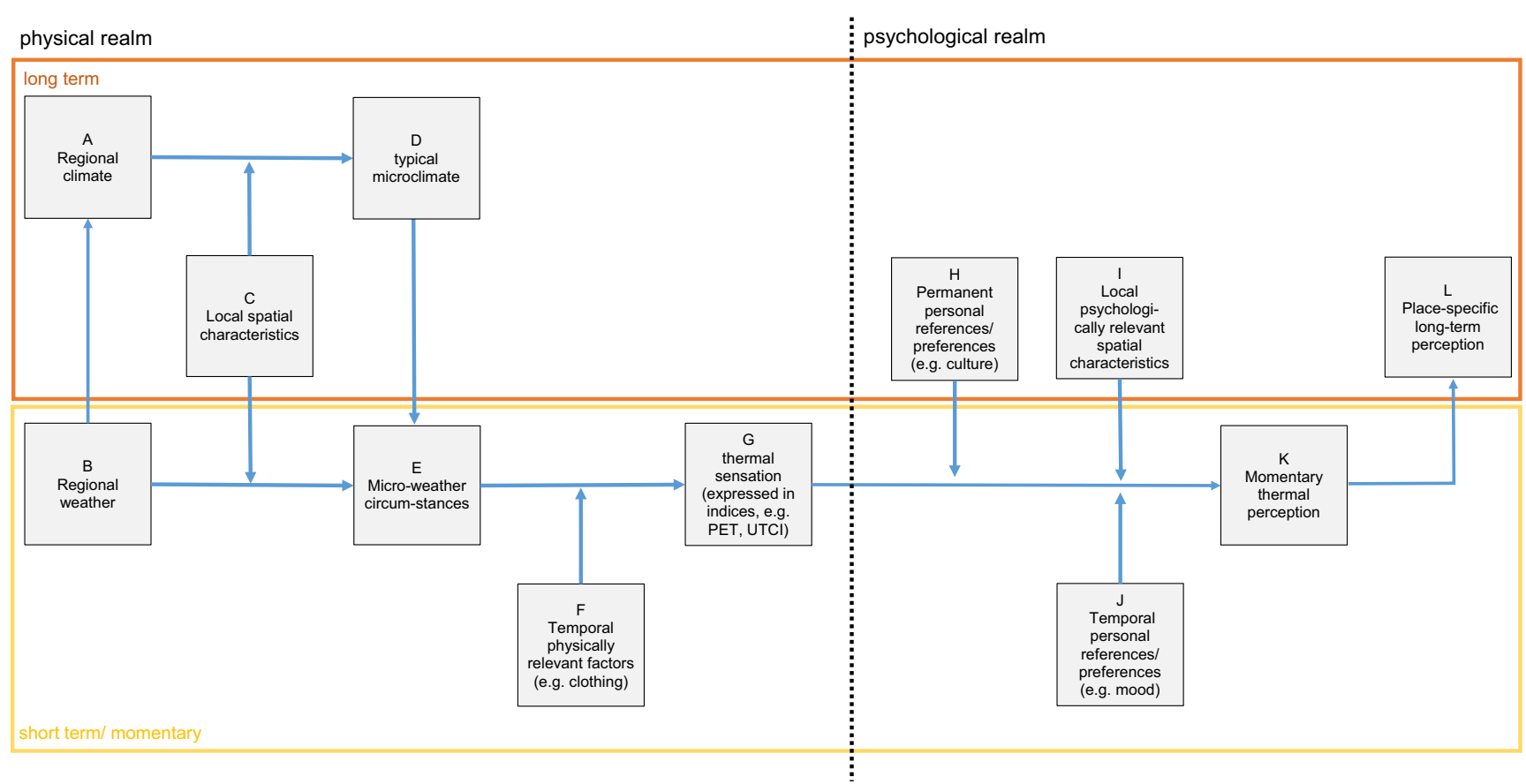

Fig. 1 The new PhyPsy model to describe thermal perception 
(PMV), initially developed by Fanger for indoor situations (Fanger 1970), physiologically equivalent temperature (PET) that has been broadly used in Europe (Mayer and Höppe 1987; Matzarakis et al. 1999), the COMFA model that is often used in North America (Brown and Gillespie 1986; Vanos et al. 2012), and recently the universal thermal climate index (UTCI) that takes into account a wider range of physiological factors than the other indices and that is geared at a worldwide ubiquitous use (Fiala et al. 2012; Jendritzky et al. 2012). More indices for outdoor thermal perception are described in Potchter et al. (2018).

During the past two decades, the purely physical/ physiological approach has been questioned, based on earlier findings from indoor studies. In 1981, Auliciems introduced a psychological approach to indoor studies and defined the term "thermal perception" (Auliciems 1981; Heijs and Stringer 1988) and developed the concept further into a model in which people's perceptions and preference were reflected (De Dear and Brager 1998). This approach also found its way into outdoor thermal perception studies: the physical/physiological measurements to determine thermal sensation were supplemented with interviews of people to understand the psychological aspects of thermal perception in first studies by Nikolopoulou et al. (2001). Inconsistencies appeared between the measured objective data and the subjective data and indicated that psychological factors had an influence. A similar, but up-scaled study on this topic was undertaken in the RUROS project that entailed field studies in five different European countries (Nikolopoulou and Lykoudis 2006) and in a crosscountry project in Sweden and Japan (Knez and Thorsson 2006; Thorsson et al. 2007; Knez and Thorsson 2008).

The cross-European RUROS project as well as studies by Thorsson, Knez et al. revealed that significant differences in thermal perception exist between countries based on habituation, personal backgrounds and cultural differences. More studies on outdoor thermal perception by other research groups worldwide followed suit, e.g., Andrade et al. (2011), Lin et al. (2011), and meta-study by Kántor et al. (2012)), and some authors specified these psychologically relevant factors (Lin et al. 2011; Krüger and Rossi 2011; Chen et al. 2015; Lindner-Cendrowska and Błażejczyk 2018; Lam and Lau 2018; Johansson et al. 2018; Brychkov et al. 2018; Peng et al. 2019). In all of these studies, climate habituation and resulting expectations were deemed relevant for outdoor thermal perception. In our new conceptual PhyPsy model, we summarize this as "permanent personal references and preferences" (box H). But also other "permanent personal references and preferences" than habituation were identified, and to illustrate those, we would like to discuss a few examples from the literature. Tung et al. noticed that cultural preferences for maintaining lighter skin tones have an impact on microclimate preferences (Tung et al. 2014). Aljawabra and Nikolopoulou identified socio-economic influences-such as education, job, and self-evaluation of the economic state of the intervieweeon thermal perception (Aljawabra and Nikolopoulou 2010). For instance, ownership of air-conditioned cars and houses amongst higher income groups brought about more sensitivity to climatic circumstances. Yung et al. showed that a higher economic status relates to a narrower range of what is considered acceptable in terms of thermal comfort, for the same reasons as identified by Aljawabra and Nikolopoulou (Yung et al. 2019). Another type of influence concerned people's attitudes. Knez (2005) described that people with different attitudes to "outdoor life" also have different thermal perceptions. This research showed that people who practice an outdoor lifestyle are also more accustomed to larger temperature differences and tend to accept a broader range of circumstances as thermally comfortable.

A third category of psychologically relevant factors was identified: spatial characteristics that influence the thermal perception (box I). Knez et al. gave some hints on the spatial parameters that can influence thermal perception: "form (structure, openness), material (surface characteristics), naturalness (degree of artificiality), and location (space dimension)" (Knez et al. 2009, p. 104). Although they were aware of a potential relevance of the spatial characteristics for environmental design, they did not address these characteristics more explicitly. Therefore, Lenzholzer and colleagues soon after studied specific spatial parameters in urban squares and showed that several parameters have an effect on long-term thermal perception: spatial proportions, degree of openness, and materiality such as the type of material used in facades, paving, or other urban elements (Lenzholzer and van der Wulp 2010; Lenzholzer and Koh 2010). This study was followed by Klemm and colleagues (Klemm et al. 2015a; Klemm et al. 2015b; Klemm et al. 2017) who indicated psychological effects of green elements on thermal perception that cannot be related to measurable biometeorological effects. Soon after, Cortesão studied building materials in urban plazas (Cortesão et al. 2016), and Krüger et al. thermal perception in different street typologies (Krüger 2017). Shih and colleagues studied spatial elements in Taiwanese school yards (Shih et al. 2017), Peng et al. the spatial environment in different urban Dutch public spaces (Peng et al. 2019), and Yung et al. in Hong Kong gardens (Yung et al. 2019). Also, paving material research included studies on people's visual and thermal perception of different materials (Rosso et al. 2016). In all studies mentioned above, the physical/physiological and psychological factors were investigated jointly and revealed that the spatial environment independent from its purely physical effects of the environment can influence thermal perception via other cues (e.g., visual).

The other major set of psychological factors identified in the literature were short-term influences (box $\mathrm{J}$ ) that were first identified by Nikolopoulou and Steemers (2003) and then by others (also see review by Chen and Ng 2012). These 
comprise a person's mood at a specific moment (Knez and Thorsson 2008; Yin et al. 2012; Lindner-Cendrowska and Błażejczyk 2018; Peng et al. 2019), whether or not a person is with company (Nikolopoulou and Lykoudis 2006; Sharifi et al. 2016; Peng et al. 2019), and has perceived control over the microclimatic conditions and the persons' intention for a site visit (Nikolopoulou and Lykoudis 2006; Lin 2009; Tung et al. 2014; Sharifi et al. 2016; Lindner-Cendrowska and Błażejczyk 2018; Johansson et al. 2018). Another temporary factor is "alliesthesia," meaning that the perception of a thermal stimulus can differ depending on other simultaneous sensory stimuli (De Dear 2011; Shooshtarian and Ridley 2017; Lindner-Cendrowska and Błażejczyk 2018; Yung et al. 2019).

To summarize, we hypothesize that, together with factors from the physiological/physical realm of thermal sensation, psychological factors influence momentary thermal perceptions (box K in Fig. 1). An accumulation of these momentary perceptions can lead to long-term thermal perceptions for specific places (box L in Fig. 1) and eventually be distilled into "mental schemata" for certain types of outdoor spaces as indicated by Lenzholzer (2010), in accordance with findings in the literature in environmental psychology (e.g., Pezdek et al. 1989). These "mental schemata" are also known to serve as a psychological mechanism to assess unknown situations and a similar process can be expected for the shaping of thermal perception in unknown circumstances.

\section{Identifying fields of future research and suitable methods}

There is a plethora of studies on the physical and the physiological factors of microclimate that influence people's thermal sensation (e.g., see reviews by Chen and Ng 2012; Johansson et al. 2014; Potchter et al. 2018) and the existing knowledge allows rather precise predictions of thermal sensation (see boxes A-G in Fig. 1). The knowledge generated in these fields also finds its way into thermal indices that try to describe thermal sensation in a quantitative way. A continuous refinement of the different thermal indices is taking place and generally speaking, the physical and physiological part of outdoor thermal perception is quite well-covered and much current research is devoted to these aspects. In contrast, the psychological factors have not yet been covered extensively: studies have only started recently and are often in their infancy. As this paper focuses on the psychological factors, we will cast more light on the knowledge gaps and methods for studying those aspects. In the following, we will discuss these separately, in accordance with the different concepts shown in the PhyPsy model (see Fig. 1).

Although we focus the discussion on studying the psychological factors, we would like to emphasize that each of the methods suggested below should be supplemented with collecting reliable data about the physiological/physical conditions in situ. Longitudinal spatially fine-grained measurement campaigns have to be conducted to depict the microclimate and micro-weather parallel with the perception research. But as outdoor meteorological conditions cannot be controlled, it might be difficult to assess the effect of situational aspects that are deemed psychologically relevant. Physical and physiologically relevant parameters can only be kept constant in experimental setups, i.e., under laboratory conditions. As long as the physical and physiologically relevant parameters are the same in all experimental conditions, and subjects are assigned at random to these conditions, observed differences in thermal perception may be attributed to the varying experimental factors. However, the effect of the factors that are hypothesized to be of psychological importance for thermal perception may interact with the physiologically relevant factors. So, even if these physiologically relevant factors are not experimentally varied, it is advisable to record and report their values. If interactions between physiologically and psychologically relevant factors are hypothesized, the experimenter has to be able to systematically vary (also) the physiologically relevant factors. For the physiologically relevant factors to systematically vary, an outdoor physical environment has to be simulated within the laboratory. To the extent that this simulation is experienced as more realistic by the subject, the outcomes may be ascribed more "ecological validity," i.e., the outcomes are more likely to apply to real outdoor environments as well. This parallel inquiry of physiological/physical factors allows a control, to clearly identify differences between thermal sensation and perception and to draw conclusions on the way the psychological factors modify thermal perception.

\section{Momentary references and preferences}

Studies regarding the momentary references and preferences (box J in Fig. 1) have mainly focused on people's mood, company, perceived control, and reasons for visits. Apart from these parameters, there might be factors such as the "vision-, sound-, and smellscapes" at a certain moment that might influence momentary thermal perception as well. Research on human multisensory perception and the related concept of "alliesthesia" suggests that cues from one sense can influence a perception in a different sense. For instance, the sounds of falling water can generate a "cool" atmosphere or the smell of wood fire fumes a warm "fireplace" atmosphere, although the thermal environment is unchanged. The concept of "alliesthesia" could be specified through the identification of specific cues.

To study momentary references and preferences related to thermal perception, interviews need to be applied as the parameters determining references and preferences can only be verbalized by the individual study participants. Data on these 
references and preferences cannot be derived from inferences such as observations of behavior because behavior is influenced by many other factors than the thermal perception. The interviews (structured or semi-structured) can be conducted face-to face or with a "perceptual-thermal notebook" in which respondents record their thermal perception in relation to other factors at the time they experience it (e.g., during "experience walks"). Alternatively, smartphone apps can be used to record respondent's experience. As these preferences might diverge depending on cultures, it might be advisable to conduct such studies in different cultures, with similar or different climatic conditions.

\section{Stable references and preferences}

A person's more permanent references and preferences (box $\mathrm{H}$ in Fig. 1) have only been studied in some countries (see, e.g., Brychkov et al. 2018). Other regions need to be studied to learn more about the differences in thermal perception (e.g., habituation, cultural differences). Apart from that, more sociocultural aspects need to be considered, such as the influence of income on how dwellings and outdoor spaces can be shaped and how people might experience the microclimate and, weather that these environments create. Studies about attitudes similar to the research on "outdoor lifestyle" (Knez 2005; Knez and Thorsson 2006) could cover other types of attitudes, too. These attitudes could entail how individuals relate to concepts such as "nature," "environment(alism)," or "climate." Also, personal traits such as sensory-processing sensitivity might have an influence on thermal perception (Aron et al. 2012).

Similar to studies on momentary references and preferences, people have to verbalize their perceptions. This can take place in interviews (structured or semi-structured), but can also be noted in "diaries" that cover a longer time range (as used by Helbich et al. 2014). As these references and preferences appeared to differ depending on cultures and (climatic) regions, such studies need to be conducted in more (climatic) regions featuring different cultures.

\section{Local psychologically relevant spatial characteristics}

The stable spatial environment provides a range of sensory cues (visual, tactile, auditory, and olfactory) that can relate to shortterm and indirectly to long-term thermal perception. The longterm perception and people's related mental schemata appear to be influenced by the stable spatial environment. Recent studies have only covered a few spatial parameters such as proportions of spaces (wideness, enclosure) and the environment's colors, materials/textures (hard/soft, coarse/fine). Many other spatial parameters that are widely discussed in the literature on landscape and urban design (e.g., Cullen 1971; Bacon 1974; Lynch and Hack 1984; Jakle 1987; Motloch 2001; Loidl and Bernard
2003; Vroom 2006; Gehl 2013) have not been covered. Amongst these are a space's light conditions, the types of spatial elements (earthen, built, vegetation, water), size and scale of buildings and other volumes (e.g., vegetation), the style of the spatial features (historical/modern, natural/architectonic), and order in spatial layout (rhythm/harmony/symmetry or chaotic). The fact that spatial environments can be changed comparatively easily, in contrast to people's references and preferences, makes outdoor space design potentially an effective tool to influence people's thermal perception. Hence, more research on these factors can yield very relevant knowledge for environmental design. Apart from that, outdoor space design also shapes the physical microclimate/micro-weather and we do not know how the physical and psychological effects of the environment exactly interact.

To study these questions about the relation between spatial parameters and people's perception, a wider repertoire of methods than interviews alone is needed. Various "immersive experiences" in different types of spaces can be employed in which visual, tactile, auditory, and olfactory cues can be related to thermal perception, building further on the initial study of Vigier et al. (2015). These "immersive experiences" experiments can take place in carefully selected spatial typologies (e.g., very different street profiles or green spaces, also see Stigsdotter et al. 2017) in augmented reality settings or in virtual environments (Krüger and Costa 2019). As real-life environments are often hard to shape according to a quasiexperimental setup, mock-ups of certain spatial configurations may be used instead. Obviously, these simulated environments can only mimic reality to some degree and in experiments using such techniques simultaneous inquiries need to be conducted to validate the simulative power of these environments.

Within selected real spaces, social value mapping may prove to be a useful method. In social value mapping, people indicate locations on a map that they deem to have certain values or where specific conditions are strongly present/ occur frequently or are absent. A relatively new method for real-life settings is having people perform assessments with a smart phone app during a longer period of time. The use of the smart phone with built-in GPS capabilities makes this method spatially explicit without that the participant having to specify the location him-/or herself. The same is true for the date- and timestamp. So, time and location are known (although GPS accuracy may not always be very high). Participants can be prompted to fill out a short questionnaire by a push message. Prompts can be according to a random time sampling method. However, this is likely to generate many responses while participants are indoors. In order to increase the number of prompts at moments that the participant is outdoors (and likely to respond), it is perhaps better to send push messages at times when the GPS measurements provided by the smart phone indicate that the participant is walking. 
Virtual reality techniques also seem promising (Kuliga et al. 2015). A cave automatic virtual environment (CAVE), with subjects wearing $3 \mathrm{D}$ glasses, is an option to simulate visual and audible (possibly even olfactory) cues that might have an impact on thermal perception. Obviously, in such a context, the thermal environment (air temperature, ventilation, etc.) needs to be manipulable to test the physiological effects and compare these to psychological effects. New digital techniques may also be helpful to do research in real-life outdoor setting. In that case, augmented reality techniques offer new opportunities. Subjects are wearing special glasses or a headmounted device and virtual changes in the environment are superimposed on the real physical environment. This technique also allows for systematic manipulation of the physical environment. Augmented reality can also be offered by a smart phone camera and display. However, the level of immersion is likely to be much lower in such cases. The subject is responding more to a scenario than actually experiencing the scenario: if the environment looked like this, how would you rate the thermal conditions? Similar challenges may be posed by techniques using photographs or photorealistic simulations (as used in Tang et al. 2016 or Arnberger et al. 2017) to inquire about thermal perception.

\section{Other fields of future research}

The relations between the momentary thermal experience (box K) and the long-term thermal perception (box L) also require more research. Lenzholzer (2010) addressed this issue in only one study that showed that situations with high winds seem to be engrained in people's memory and shape mental schemata, but it was not possible to draw conclusions about how this exactly happens. It would be necessary to know how patterns of recurrence of certain micro-weather experiences in certain spaces influence long-term perception. This would involve longitudinal studies including local measurements of micro-weather, interviews that cover momentary experiences, and long-term perceptions with the same respondents. Also, the relations between the recently identified parameters on psychological factors (boxes $\mathrm{H}-\mathrm{L}$ ) should be studied in more detail, for instance, by creating more extensive questionnaires.

Eventually it should be possible to establish an understanding of the ratio of psychological and physical factors in thermal perception and to quantify the influence of the different factors we described in the PhyPsy model (Fig. 1). Such quantifications could help to generate algorithms for the psychological factors which can then be embedded in agent-based-modeling (Elsawah et al. 2015) of thermal perception. For the physical/physiological aspects, such models already exist (e.g., BOT-world). Including psychological factors in such models would provide a more precise prediction of thermal perception in specific environmental settings. Yet, to be able to shape such models, much research on the environmental psychological factors is needed to be able to eventually build a solid evidence basis.

\section{Concluding remark: Where to start?}

It will take many more studies in different climates, regions, and cultures to be able to understand the psychological side of thermal perception in a more precise way and it is necessary to conduct more comparative research on the related physiological/physical factors, too. This requires collection of micro-weather data, and to create reliable data, the studies need to be rather longitudinal. Hence, it will take some more years of research to arrive at a state of the art where a quantification of the influences of the physiological/physical and the psychological factors is possible.

However, to provide useful knowledge for outdoor space design in specific climates, regions, and cultures, such detailed worldwide comparative studies on all factors of the PhyPsy model are not necessary. Because spatial factors have an effect on the physical, physiological, and the psychological aspects, we suggest to first conduct more studies on the spatial factors of the outdoor environment. Priority should be given to studies in the most problematic and populated regions of the world such as Southeast Asia, Africa, and South America. Research on these factors will yield relevant and useful evidence for shaping human environments that will be thermally comfortable in a world challenged by the impacts of climate change.

Open Access This article is distributed under the terms of the Creative Commons Attribution 4.0 International License (http:// creativecommons.org/licenses/by/4.0/), which permits unrestricted use, distribution, and reproduction in any medium, provided you give appropriate credit to the original author(s) and the source, provide a link to the Creative Commons license, and indicate if changes were made.

\section{References}

Aljawabra F, Nikolopoulou M (2010) Influence of hot arid climate on the use of outdoor urban spaces and thermal comfort: do cultural and social backgrounds matter? Intelligent Build Int 2(3):198-217

Andrade H, Alcoforado M-J, Oliveira S (2011) Perception of temperature and wind by users of public outdoor spaces: relationships with weather parameters and personal characteristics. Int J Biometeorol 55(5):665-680. https://doi.org/10.1007/s00484-010-0379-0

Arnberger A, Allex B, Eder R, Ebenberger M, Wanka A, Kolland F, Wallner P, Hutter H-P (2017) Elderly resident's uses of and preferences for urban green spaces during heat periods. Urban For Urban Green 21:102-115

Aron EN, Aron A, Jagiellowicz J (2012) Sensory processing sensitivity: a review in the light of the evolution of biological responsivity. Personal Soc Psychol Rev 16(3):262-282. https://doi.org/10.1177/ 1088868311434213

Auliciems A (1981) Towards a psycho-physiological model of thermal perception. Int J Biometeorol 25(2):109-122

Bacon EN (1974) Design of Cities. Viking Press, New York

Blocken B, Carmeliet J (2004) Pedestrian wind environment around buildings: literature review and practical examples. J Therm Envel Build Sci 28(2):107-159 
Brown RD, Gillespie T (1986) Estimating outdoor thermal comfort using a cylindrical radiation thermometer and an energy budget model. Int J Biometeorol 30(1):43-52

Brown RD, Gillespie TJ (1995) Microclimatic landscape design : creating thermal comfort and energy efficiency. Wiley, New York

Brychkov D, Garb Y, Pearlmutter D (2018) The influence of climatocultural background on outdoor thermal perception. Int $\mathrm{J}$ Biometeorol 62(10):1873-1886

Chen L, Ng E (2012) Outdoor thermal comfort and outdoor activities: a review of research in the past decade. Cities 29(2):118-125

Chen L, Wen Y, Zhang L, Xiang W-N (2015) Studies of thermal comfort and space use in an urban park square in cool and cold seasons in Shanghai. Build Environ 94:644-653. https://doi.org/10.1016/j. buildenv.2015.10.020

Cheung PK, Jim CY (2018) Subjective outdoor thermal comfort and urban green space usage in humid-subtropical Hong Kong. Energy and Buildings 173:150-162. https://doi.org/10.1016/j.enbuild.2018. 05.029

Cortesão J, Alves FB, Corvacho H, Rocha C (2016) Retrofitting public spaces for thermal comfort and sustainability. Indoor and Built Environment 25(7):1085-1095. https://doi.org/10.1177/ $1420326 \times 16659326$

Cullen G (1971) The concise townscape. Routledge, London

De Dear R (2011) Revisiting an old hypothesis of human thermal perception: alliesthesia. Build Res Inf 39(2):108-117

De Dear R, Brager GS (1998) Developing an adaptive model of thermal comfort and preference

D'Ippoliti D, Michelozzi P, Marino C, De'Donato F, Menne B, Katsouyanni K, Kirchmayer U, Analitis A, Medina-Ramón M, Paldy A (2010) The impact of heat waves on mortality in 9 European cities: results from the EuroHEAT project. Environ Health 9(1):37

Elsawah S, Guillaume JH, Filatova T, Rook J, Jakeman AJ (2015) A methodology for eliciting, representing, and analysing stakeholder knowledge for decision making on complex socio-ecological systems: from cognitive maps to agent-based models. J Environ Manag 151:500-516

Fanger PO (1970) Thermal comfort : analysis and applications in environmental engineering. Copenhagen

Fiala D, Havenith G, Bröde P, Kampmann B, Jendritzky G (2012) UTCIFiala multi-node model of human heat transfer and temperature regulation. Int J Biometeorol 56(3):429-441

Field CB (2014) Climate change 2014-impacts, adaptation and vulnerability: regional aspects. Cambridge University Press, Cambridge

Gehl J (2013) Cities for people. Island press, Washigton DC

Gosling SN, Lowe JA, McGregor GR, Pelling M, Malamud BD (2009) Associations between elevated atmospheric temperature and human mortality: a critical review of the literature. Clim Chang 92(3-4): 299-341

Haines A, Kovats RS, Campbell-Lendrum D, Corvalan C (2006) Climate change and human health: impacts, vulnerability and public health. Public Health 120(7):585-596

Heijs W, Stringer P (1988) Research on residential thermal comfort: some contributions from environmental psychology. J Environ Psychol 8(3):235-247

Helbich M, Böcker L, Dijst M (2014) Geographic heterogeneity in cycling under various weather conditions: evidence from Greater Rotterdam. J Transp Geogr 38:38-47

Humphreys MA, Hancock M (2007) Do people like to feel 'neutral'?: exploring the variation of the desired thermal sensation on the ASHRAE scale. Energy Build 39(7):867-874. https://doi.org/10. 1016/j.enbuild.2007.02.014

Jacobs CMJ, Elbers JA, Brolsma R, Hartogensis OK, Moors EJ, Rodríguez-CarreteroMárquez MT, Hove B (2015) Assessment of evaporative water loss from Dutch cities. Build Environ 83:27-38
Jakle JA (1987) The visual elements of landscape. Univ of Massachusetts Press

Jendritzky G, Dear R, Havenith G (2012) UTCI — why another thermal index? Int J Biometeorol 56(3):421-428. https://doi.org/10.1007/ s00484-011-0513-7

Johansson E, Thorsson S, Emmanuel R, Krüger E (2014) Instruments and methods in outdoor thermal comfort studies - the need for standardization. Urban Clim 10(Part 2):346-366. https://doi.org/10.1016/j. uclim.2013.12.002

Johansson E, Yahia MW, Arroyo I, Bengs C (2018) Outdoor thermal comfort in public space in warm-humid Guayaquil, Ecuador. Int $\mathrm{J}$ Biometeorol 62(3):387-399. https://doi.org/10.1007/s00484-0171329 -x

Kántor N, Unger J, Gulyás Á (2012) Subjective estimations of thermal environment in recreational urban spaces - part 2: international comparison. Int J Biometeorol 56(6):1089-1101. https://doi.org/ 10.1007/s00484-012-0564-4

Klemm W, Heusinkveld BG, Lenzholzer S, Jacobs MH, Van Hove B (2015a) Psychological and physical impact of urban green spaces on outdoor thermal comfort during summertime in the Netherlands. Build Environ 83:120-128

Klemm W, Heusinkveld BG, Lenzholzer S, van Hove B (2015b) Street greenery and its physical and psychological impact on thermal comfort. Landsc Urban Plan 138:87-98

Klemm W, van Hove B, Lenzholzer S, Kramer H (2017) Towards guidelines for designing parks of the future. Urban For Urban Green 21: 134-145

Knez I (2005) Attachment and identity as related to a place and its perceived climate. J Environ Psychol 25(2):207-218. https://doi.org/ 10.1016/j.jenvp.2005.03.003

Knez I, Thorsson S (2006) Influences of culture and environmental attitude on thermal, emotional and perceptual evaluations of a public square. Int J Biometeorol 50(5):258-268. https://doi.org/10.1007/ s00484-006-0024-0

Knez I, Thorsson S (2008) Thermal, emotional and perceptual evaluations of a park: cross-cultural and environmental attitude comparisons. Build Environ 43(9):1483-1490. https://doi.org/10.1016/j. buildenv.2007.08.002

Knez I, Thorsson S, Eliasson I, Lindberg F (2009) Psychological mechanisms in outdoor place and weather assessment: towards a conceptual model. Int J Biometeorol 53(1):101-111. https://doi.org/10. 1007/s00484-008-0194-Z

Kovats RS, Hajat S (2008) Heat stress and public health: a critical review. Annu Rev Public Health 29:41-55

Krüger E (2017) Impact of site-specific morphology on outdoor thermal perception: a case-study in a subtropical location. Urban Clim 21: 123-135. https://doi.org/10.1016/j.uclim.2017.06.001

Krüger EL, Costa T (2019) Interferences of urban form on human thermal perception. Sci Total Environ 653:1067-1076

Krüger EL, Rossi FA (2011) Effect of personal and microclimatic variables on observed thermal sensation from a field study in southern Brazil. Build Environ 46(3):690-697. https://doi.org/10.1016/j. buildenv.2010.09.013

Kuliga SF, Thrash T, Dalton RC, Hölscher C (2015) Virtual reality as an empirical research tool - exploring user experience in a real building and a corresponding virtual model. Comput Environ Urban Syst 54: 363-375

Lam CKC, Lau KK-L (2018) Effect of long-term acclimatization on summer thermal comfort in outdoor spaces: a comparative study between Melbourne and Hong Kong. Int J Biometeorol 62(7): 1311-1324. https://doi.org/10.1007/s00484-018-1535-1

Lenzholzer S (2010) Engrained experience-a comparison of microclimate perception schemata and microclimate measurements in Dutch urban squares. Int J Biometeorol 54(2): 141-151

Lenzholzer S (2015) Weather in the City - how design shapes the urban climate. nai010 publishers, Rotterdam 
Lenzholzer S, Koh J (2010) Immersed in microclimatic space: microclimate experience and perception of spatial configurations in Dutch squares. Landsc Urban Plan 95:1-15

Lenzholzer S, van der Wulp NY (2010) Thermal experience and perception of the built environment in Dutch urban squares. J Urban Des 15(3):375-401

Lin T-P (2009) Thermal perception, adaptation and attendance in a public square in hot and humid regions. Build Environ 44(10):2017-2026. https://doi.org/10.1016/j.buildenv.2009.02.004

Lin T-P, de Dear R, Hwang R-L (2011) Effect of thermal adaptation on seasonal outdoor thermal comfort. Int J Climatol 31(2):302-312. https://doi.org/10.1002/joc. 2120

Lindner-Cendrowska K, Błażejczyk K (2018) Impact of selected personal factors on seasonal variability of recreationist weather perceptions and preferences in Warsaw (Poland). Int J Biometeorol 62(1):113 125. https://doi.org/10.1007/s00484-016-1220-1

Loidl H, Bernard S (2003) Opening spaces : design in landscape architecture. Birkhäuser, Basel

Lynch K, Hack G (1984) Site planning. MIT press

Matzarakis A, Mayer H, Iziomon MG (1999) Applications of a universal thermal index: physiological equivalent temperature. Int $\mathrm{J}$ Biometeorol 43(2):76-84

Mayer H, Höppe P (1987) Thermal comfort of man in different urban environments. Theor Appl Climatol 38(1):43-49

Motloch JL (2001) Introduction to landscape design. Wiley, New York

Nikolopoulou M, Lykoudis S (2006) Thermal comfort in outdoor urban spaces: analysis across different European countries. Build Environ 41(11):1455-1470

Nikolopoulou M, Steemers K (2003) Thermal comfort and psychological adaptation as a guide for designing urban spaces. Energy and Buildings 35(1):95-101

Nikolopoulou M, Baker N, Steemers K (2001) Thermal comfort in outdoor urban spaces: understanding the human parameter. Sol Energy 70(3):227-235

Oke TR, Mills G, Christen A, Voogt JA (2017) Urban climates. Cambridge University Press, Cambridge

Parsons KC (2003) Human thermal environments : the effects of hot, moderate, and cold environments on human health, comfort, and performance. In: London, 2nd edn. Taylor \& Francis, New York

Peng Y, Feng T, Timmermans H (2019) A path analysis of outdoor comfort in urban public spaces. Build Environ 148:459-467. https://doi. org/10.1016/j.buildenv.2018.11.023

Pezdek K, Whetstone T, Reynolds K, Askari N, Dougherty T (1989) Memory for real-world scenes: the role of consistency with schema expectation. J Exp Psychol Learn Mem Cogn 15(4):587-595

Potchter O, Cohen P, Lin T-P, Matzarakis A (2018) Outdoor human thermal perception in various climates: a comprehensive review of approaches, methods and quantification. Sci Total Environ 631-632: 390-406. https://doi.org/10.1016/j.scitotenv.2018.02.276
Rosso F, Pisello AL, Cotana F, Ferrero M (2016) On the thermal and visual pedestrians' perception about cool natural stones for urban paving: a field survey in summer conditions. Build Environ 107: 198-214

Sharifi E, Sivam A, Boland J (2016) Resilience to heat in public space: a case study of Adelaide, South Australia. J Environ Plan Manag 59(10):1833-1854. https://doi.org/10.1080/09640568.2015. 1091294

Shih W-M, Lin T-P, Tan N-X, Liu M-H (2017) Long-term perceptions of outdoor thermal environments in an elementary school in a hothumid climate. Int J Biometeorol:1-10

Shooshtarian S, Ridley I (2017) The effect of physical and psychological environments on the users thermal perceptions of educational urban precincts. Build Environ 115:182-198. https://doi.org/10.1016/j. buildenv.2016.12.022

Stigsdotter UK, Corazon SS, Sidenius U, Refshauge AD, Grahn P (2017) Forest design for mental health promotion - using perceived sensory dimensions to elicit restorative responses. Landsc Urban Plan 160:1-15

Tang H, Klemm W, Lenzholzer S (2016) Design guidelines for thermally comfortable and attractive streetscapes in harbour areas. Adapation Futures 2016. Rotterdam

Thorsson S, Honjo T, Lindberg F, Eliasson I, Lim EM (2007) Thermal comfort and outdoor activity in Japanese urban public places. Environ Behav 39(5):660-684. https://doi.org/10.1177/ 0013916506294937

Tung C-H, Chen C-P, Tsai K-T, Nm K, Hwang R-L, Matzarakis A, Lin T$P$ (2014) Outdoor thermal comfort characteristics in the hot and humid region from a gender perspective. Int J Biometeorol 58(9): 1927-1939. https://doi.org/10.1007/s00484-014-0795-7

Vanos JK, Warland JS, Gillespie TJ, Kenny NA (2012) Thermal comfort modelling of body temperature and psychological variations of a human exercising in an outdoor environment. Int $\mathrm{J}$ Biometeorol 56(1):21-32. https://doi.org/10.1007/s00484-010-0393-2

Vigier T, Moreau G, Siret D (2015)From visual cues to climate perception in virtual urban environments. In: 2015 IEEE Virtual Reality (VR), IEEE, pp 305-306

Vroom MJ (2006) Lexicon of garden and landscape architecture. Mato J. Vroom. Basel: Birkhäuser,

Westerberg U, Knez I, Eliasson I (2003) Urban climate spaces. In: The 5 th international conference on urban climate (ICUC-5). Lodz, Poland, p 2003

Yin J, Zheng Y, Wu R, Tan J, Ye D, Wang W (2012) An analysis of influential factors on outdoor thermal comfort in summer. Int $\mathrm{J}$ Biometeorol 56(5):941-948

Yung EHK, Wang S, Chau C-K (2019) Thermal perceptions of the elderly, use patterns and satisfaction with open space. Landsc Urban Plan 185:44-60. https://doi.org/10.1016/j.landurbplan.2019.01.003 